\title{
The symptoms and treatment of Zika virus infection
}

\author{
Siyi Zheng ${ }^{1}$ \\ ${ }^{1}$ College of Biology, Hunan University, Changsha 410082, China
}

\begin{abstract}
Zika virus is an arbovirus of the Flaviviridae family that causes a mosquito-borne disease. The infection can cause devastating complications among individuals in all age ranges, such as microcephaly in infants and Guillain-Barre syndrome in adults. Its teratogenic potential and explosive epidemics has already caused a panic and is a public health emergency. This review puts emphasis on the current acknowledge of the transmission, clinical characteristics, pathogenic mechanism, and treatment of Zika virus infection, and the future expectation to this disease as well.
\end{abstract}

\section{Introduction}

Zika virus, a flavivirus, has emerged and caused major epidemics in recent years. The virus was identified first in 1947 in Rhesus monkeys in the Zika forest in Uganda, and transmitted mainly by mosquitoes and other transmission such as sexual intercourse and mother-to-child transmission. During the past decades, Zika virus epidemic has taken place several times and affected millions of people.

The presentation of Zika fever is similar to those infections caused by Dengue and Chikungunya, which means it's nonspecific and thus might result to misdiagnosis. As all of them might present the alike symptoms in most patients such as acute fever, skin rash, conjunctivitis, and with subtle differences among them.

This is the first time in nearly half a century that an infectious disease related to human birth defects has been found, causing global panic. The most devastating disease of Zika virus infection is congenital malformations, especially microcephaly. And in adults, Zika virus infection is related to Guillain-Barre syndrome (GBS), which is an autoimmune disease leading to neurological complications.

Laboratory diagnosis is crucial during the outbreak of Zika virus, the criterion standard mostly relies on the demonstration of the virus in body fluids through RT-PCR, which can detect the viral RNA. Nevertheless, the accuracy of this method only maintains for about 1 week as the viremia is generally transient.

The outbreak of Zika virus and its potential threat make the development of preventative vaccines and therapeutic drugs a global priority. It remains no specific medicine for tackling this disease, so the prevention and surveillance of high risk areas appear to be particularly important. High-risk populations like pregnancy should avoid traveling to epidemic areas and use physical methods to prevent mosquito bites. And the prime control measures focus on the controlling of vectors, which mainly refers to mosquitoes.

\section{The discovery of Zika virus}

Zika virus was first discovered in 1947 in $\operatorname{Uganda}^{[1]}$ where scientists isolated a virus from Rhesus monkeys for yellow fever monitoring and called it 'Zika virus'. Since $1952^{[2]}$, human beings infected with Zika virus were found in Uganda and Tanzania, and no large-scale epidemic had been formed until $2007^{[3]}$, when the epidemics of Zika virus had begun to break out in Micronesia Federal..

Like Yellow fever virus, Japanese encephalitis virus, Dengue virus, and West Nile virus, Zika virus is also a mosquito-borne flavivirus. At first, there was no indication that the Zika virus could cause human disease. It was until 1953 human illness caused by Zika virus had been recognized in Nigeria ${ }^{4}$ with a mild and febrile illness as symptoms. The outbreak in French Polynesia ${ }^{5}$ in 2013 and 2014 might have involved about 32,000 people, except the condition similar to the above, cases of Guillain-Barre syndrome were also been observed. In 2016, French Polynesian investigators identified an increased number of microcephaly, which is a kind of fetal abnormalities, after the outbreak of Zika virus in the country ${ }^{6}$. Since then, such severe neurological complications were suspected to be associated with Zika virus.

\section{Infection mechanism}

\subsection{Transmission}

Patients, asymptomatic carrier and nonhuman primates infected with ZIKV can be the source of infection. The population is generally susceptible. Several mosquito species can transmit the virus through their bite, mainly belonging to the stegomyia and diceromyia subgenera of aedes are probably enzootic vectors in Africa and Asia,

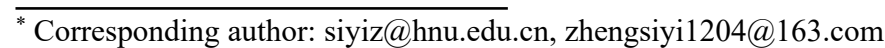


which includes A.africanus, A.furcifer, A.luteocephalus, and A.taylori ${ }^{7}$. Zika virus transmits in a human-mosquitohuman transmission cycle. A.aegypti and A.albopictus, which belong to stegomyia subgenus of aedes, are two species linked with almost all known Zika virus outbreaks. They widely distributed throughout the tropical and subtropical world and are primarily diurnal. A.albopictus is able to extend the potential range of epidemic outbreaks as it can survive in more temperature areas than the other.

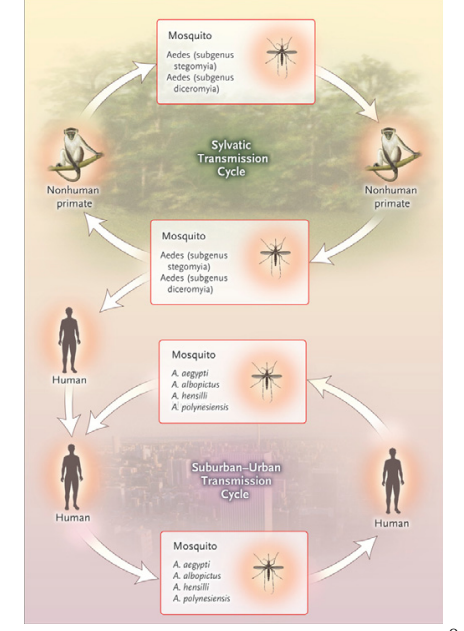

Fig1. Mosquito-borne transmission ${ }^{8}$

Besides mosquito-borne transmission, there are substantial evidence indicates that the mother can transmit Zika virus to the fetus during pregnancy, including intrauterine infections and infections during childbirth. To those fetuses who had cerebral abnormalities, Zika virus RNA can be identified in the amniotic fluid of their mothers 9 . And for children who were born with microcephaly and died soon after birth, viral antigen and RNA are also able to be detected in the brain tissue and placentas ${ }^{10}$.

There were reports ${ }^{11}$ showed sexual transmission to partners of returning male travels who were infected by Zika virus abroad. ZIKV was detected in semen and urine $^{12}$, so it can be transmitted by sexual intercourse and is a potentially sexually transmitted virus. Although the main mode of transmission of Zika fever is thought to be via mosquito bite, the low viremia observed in patients and the rapid spread within and among countries in a region like America suggest other modes of transmission. The evidence of sexual transmission suggests a mode of interhuman transmission that could contribute to its rapid spread.

\subsection{Clinical manifestation and complications}

Zika virus disease, also known as Zika fever, is a viral disease caused by Zika virus and transmitted mainly by mosquito. The incubation period of Zika fever is about 1 week, similar to other mosquito-borne flaviviruses. There's a case ${ }^{13}$ of a febrile illness lasting 4 days developed 82 hours after subcutaneous inoculation. The result of serosurvey showed that only $19 \%$ of persons infected by Zika virus had correlative symptoms ${ }^{14}$. The common clinical symptoms of Zika virus disease include fever, rash, conjunctivitis, arthralgia and muscle pain. The general rash is maculopapular or pruritic, while the fever is commonly short-term and low-grade. The clinical manifestations of ZIKV infection are generally mild, with symptoms lasting from several days to one week. The mortality rate is extremely low.

In addition to the common symptom, Zika virus is associated with severe neurological damage in newborns, especially microcephaly. Researches from Brazil ${ }^{15}$ have considered that some of the infants with microcephaly are connected to the infection of Zika virus and have a phenotype matching with fetal brain disruption. The RNA of Zika virus was detected in the amniotic fluid of pregnant women who gave birth to microcephalic newborns and in the brains of these infants. Microcephaly has a clinical feature of a small head size for gestational age and sex and indicating an underlying problem with the growth of the brain ${ }^{16}$. The disruption sequence in the fetal brain will has an influence after relatively normal brain development in early pregnancy, and will cause the destruction of fetal brain tissue following with collapse of the fetal skull, and finally results to microcephaly.

The infection of Zika virus can also cause neurological complications in adults, which is Guillain-Barre Syndrome $(\mathrm{GBS})^{17}$. GBS is a disease with the symptom as the body's immune system attacks the peripheral nervous system. It can affect sensory and motor nerves, resulting in weakness of limbs and sensory deficits.

\subsection{Molecule Mechanisms}

There are studies ${ }^{18}$ shows that Zika virus can infect human beings through receptors on human dermal fibroblasts, immature dendritic cells, and epidermal keratinocytes. These receptors permit the entry of Zika virus, including DCSIGN, Tyro, AXL, and TIM-1 entry/adhesion factors. The replication of Zika virus can activate an antiviral immune response in infected cells and promote the production of type I interferon as well. In the infected fibroblasts, the infection may also induce an autophagous program which is confirmed by the presence of characteristic autophagosome-like vesicles. $\mathrm{T}$ cells, during the acute stage of Zika fever, are activated.

Among the various complications, microcephaly is an urgent global health concern. The direct target cells of Zika virus in the developing fetus remains unknown. There is research ${ }^{19}$ suggests that Zika virus infects human cortical neural progenitor cells (hNPCs) as a potential target directly with high efficiency, and cause stunted growth of cell population and transcriptional dysregulation. Zika virus infection attenuate hNPC growth through increasing cell death and dysregulating cell-cycle progression, and the infected cells furhter release infectious Zika virus particles.

Recently another research ${ }^{20}$ has come up with new ideas that Zika virus can degrade the $\omega-3$ fatty acid transporter Mfsd2a (major facilitator superfamily domain-containing protein 2) which locates in brain microvascular endothelial cells and thus impairs lipid homeostasis. The proper brain development needs a homeostatic environment which is provided by neurovascular function and Mfsd2a is essential in 
mediating the brain uptake of DHA (docosahexaenoic acid). DHA is a kind of $\omega-3$ fatty acid which is critical for normal brain growth and cognitive function. The researchers have found that the pathogenesis of Zika virus is closely related to Mfsd2a as the Zika virus envelope protein can interact with Mfsd2a specifically and promote its polyubiquitination for proteasome-dependent degradation. Therefore the infection of Zika virus can cause obvious differences in DHA-containing in lipidomic analysis. This finding may has a potential therapeutic option for Zika virus infection that the supplementation with DHA might be able to rescue the growth restriction and microcephaly.

\section{Treatment}

\subsection{Diagnosis}

The detection of viral nucleic acid by RT-PCR and detection of IgM antibodies by MAC-ELISA (IgMcapture enzyme-linked immunosorbent assay) are the mainstays of diagnosis of Zika virus infection. Although viral nucleic acid detection can provide a definitive diagnosis, viremia is mostly transient and diagnosis is more accurate within 1 week after the onset of clinical illness ${ }^{21}$.

Additionally, viral isolation from clinical samples can be difficult as viremia is commonly low level. There's another challenge might effect the serologic test result, which is cross-reactivity of flavivirus antibodies, such as the same positive MAC-ELISA result for dengue as Zika virus infection.

\subsection{Cure}

Similar to other mosquito-borne flaviviruses, symptom is the focus in treatment for uncomplicated Zika virus infection. The need for effective vaccines against Zika virus has been highlighted all the time as Zika virus infection has causal association with microcephaly and congenital malformations in infants, and GBS in adults. Research $^{22}$ focused on Zika virus NS1, a vaccine immunogen, has been studied to explore its ability in circumventing the risk of antibody-dependent enhancement of Zika virus infection. The researchers describe a novel DNA vaccine which encodes a secreted Zika virus NS1, and can provide rapid protection from systemic Zika virus infection, thus novel NS1 T cell epitopes in vivo have been identified and result to the critical function of NS1-specific T cell response in protection against Zika virus infection.

It has been identified that integrin $\alpha v \beta 5$ can act as a promising therapeutic target for prophylactic use or treatments for Zika virus infections ${ }^{23}$. The expression of $\alpha v \beta 5$ is related to Zika virus susceptibility in various cells and tropism in human cerebral cortex development. The researchers identified integrin $\alpha v \beta 5$ as an intemalization factor for Zika virus through performing a CRISPERCas9 genome-wide screen in glioblastoma stem cells. Zika virus can bind to cells but when treated with integrin $\alpha v \beta 5$-blocking antibody, it fails to internalize, which means its infection has been inhibited. $\alpha v \beta 5$ is able to bind to Zika virus virions directly and activates the focal adhesion kinase, which is critical in Zika virus infection. Thus $\alpha v \beta 5$ blocking antibody may reduce Zika virus infection and may alleviate the pathology in human neural stem cells.

\subsection{Prevention}

There is no accomplished Zika virus vaccine yet, thus the main prevention and control measures emphasizing on avoiding mosquito bites, especially controlling the mosquito vector, and reducing sexual transmission. Potentially effective methods of prevention include avoiding travel to areas of ongoing Zika virus epidemic, avoiding unprotected sexual contact with partners at risk of Zika virus infection, and using physical means such as mosquito repellent, bed nets, and window screens ${ }^{22}$. Another way to control the vector effectively is an integrated approach which involves elimination of mosquito breeding sites and application of larvicides and insecticides as well. However, these approaches have substantial limitation, partly because the presence of cryptic breeding sites in urban settings ${ }^{25}$. In view of this, it is more suitable to combine the integrated prevention and control approach with illness detection and information communication, and development of a rapid response which involves the community as well ${ }^{26}$. Surveillance should be enhanced to monitor the impact of Zika virus on public health for potential neurological and autoimmune complications, and follow up pregnant woman to identify risk factors associated with Zika virus infection.

\section{Conclusion}

It is difficult to gauge the incidence of Zika virus infection as the symptoms are generally nonspecific and mild. Flavivirus antibody cross-reactivity makes serologic assessment complicated in areas ongoing dengue epidemic in laboratory diagnosis. The underlying reasons for the appearance of Zika virus all over the world in the past decade remain unknown. Recent global increase in the incidence and spread of flavivirus such as Zika virus may have globalization and urbanization as the underlying mechanisms ${ }^{27}$. It is clear that we need to address identified research gaps rapidly and systematically as the long-term outlook with regard to the current Zika virus is uncertain and we have to prepare for the possible epidemic. So a complete understanding of clinical outcomes and the development of discriminating diagnostic tools for Zika virus, and new vector control products and strategies, and effective therapeutics and vaccines are of considerable importance for the long-term protection against the disease. 


\section{REFERENCES}

1. Dick GW, Kitchen SF, Haddow AJ(1952). Zika virus. I. Isolations and serological specificity[J]. Trans R Soc Trop Med Hyg, 46(5): 509-520.

2. Dick GW(1952). Zika virus. II. Pathogenicity and physical properties[J]. Trans R Soc Trop Med Hyg, 46(5): 521-534.

3. Grard G, Melanie C, Illich Manfred M, et al(2014). Zika virus in Gabon (Central Africa)-2007: a new thereat from Aedes albopictus?[J]. PLoS Negl Trop Dis, 8(2): e2681.

4. MacNamara FN(1954). Zika virus: a report on three cases of human infection during an epidemic of jaundice in Nigeria[J]. Trans R Soc Trop Med Hyg, 48: 139-145.

5. Oehler E, Watrin L, Larre P,et al(2014). Zika virus infection complicated by Guillain-Barre syndrome case report, French Polynesia, December 2013. Euro Surveill, 19, 9: 20720.

6. Cauchemez S, Besnard M, Bompard P, et al(2016). Association between Zika virus and microcephaly in French Polynesia, 2013- 15: a retrospective study[J]. Lancet, 387: 2125-2132.

7. Diallo D, Sall AA, Diagne CT, et al(2014). Zika virus emergence in mosquitoes in southeastern Senegal, 2011[J]. PLoS One, 9(10): e109442.

8. Petersen LR, Jamieson DJ, Powers AM, wt al(2016). Zika Virus[J]. New England Journal of Medicine, 374(16): 1552-1563.

9. Schuler-Faccini L, Ribeiro EM, Feitosa IM, et al(2016). Possible association between Zika virus infection and microcephaly-Brazil,2015[J]. MMWR Morb Mortal Wkly Rep, 65(3): 59-62.

10. Roosecelis B.M., Julu B, Keating M.K., et al(2016). Notes from the field: evidence of Zika virus infection in brain and placental tissues from two congenitally infected newborns and two fetal losses-Brazil, 2015[J]. MMWR Morb Mortal Wkly Rep, 65: 159160.

11. Susan L.H., Kate R, Morgan H, et al(2016). Transmission of Zika virus through sexual contact with travelers to areas of ongoing transmissioncontinental United States, 2016[J]. MMWR Morb Mortal Wkly Rep, 65: 215-216.

12. Moreira J, Peixoto TM, Machado de Siqueira A, et al(2017). Sexually acquired Zika virus: a systematic review $[\mathrm{J}]$. Clin microbiol infect, pii: S1198743X(16)30659-0.

13. Bearcroft WG(1956). Zika virus infection experimentally induced in a human volunteer[J]. Trans R Soc Trop Med Hyg, 50: 442-448.

14. Duffy MR, Chen T-H, Hancock WT, et al(2009). Zika virus outbreak on Yap Island, Federated States of Micronesia[J]. N Engl J Med, 360: 2536-2543.

15. Mlakar J, Korva M, Tul N, et al(2016). Zika virus associated with microcephaly[J]. N Engl J Med, 374: 951-958.
16. Woods CG, Parker A.(2013) Investigating microcephaly[J]. Arch Dis Child, 98: 707-713.

17. World Health Organization(2016). Identification and management of Guillain-Barre syndrome in the context of Zika virus[EB/OL].

18. Hamel R, Dejarnac O, Wichit S, et al(2015). Biology of Zika virus infection in human skin cells[J]. J Virol, 89: 8880-8896.

19. Musso D, Cao-Lormeau VM, Gubler DJ(2015). Zika virus: following the path of dengue and chikungunya?[J]. Lancet, 386: 243-244.

20. Jia Zhou, Xiaojing Chi, Min Cheng, et al(2019). Zika virus degrades the $\omega-3$ fatty acid transporter Mfsd2a in brain microvascular endothelial cells and impairs lipid homeostasis[J]. Sci. Adv, 5: eaax7142.

21. Lanciotti RS, Kosoy OL, Laven JJ, et al(2008). Genetic and serologic properties of Zika virus associated with an epidemic, Yap State, Micronesia, 2007[J]. Emerg Infect Dis, 14: 1232-1239.

22. Hengli T, Christy H, Sarah C.O, et al(2016). Zika virus infects human cortical neural progenitors and attenuates their growth[J]. Cell Stem, 18: 587-590.

23. Shaobo Wang, Qiong Zhang, Shashi Kant Tiwari, et al(2020). Integrin av $\beta 5$ Internalizes Zika Virus during Neural Stem Cells Infection and Provides a Promising Target for Antiviral Therapy[J]. Cell Reports, 30: 1-15.

24. Grubor-Bauk B, Wijesundara D.K., Masavuli M, et al(2019). NS1 DNA vaccination protects against Zika infection through T-cell mediated immunity in immunocompetent mice[J]. Sci Adv, 5: eaax2388.

25. Barrera R, Amador M, Diaz A, Smith J, MunozJordan JL, Rosario Y(2008). Unusual productivity of Aedes aegypti in septic tanks and its implications for dengue control[J]. Med Vet Entomol, 22: 62-69.

26. Manrique-Saide $\mathrm{P}$, Che-Mendoza A, Barrera-Perez $M$, et $\mathrm{al}(2015)$. Use of insecticide-treated house screens to reduce infestations of dengue virus vectors, Mexico[J]. Emerg Infect Dis, 21: 308-311.

27. Adalja AA, Sell TK, Bouri N, Franco C(2012). Lessons learned during dengue outbreaks in the United States, 2001-2011[J]. Emerg Infect Dis, 18: 608-614.

28. Hygino da Cruz Jr L.C., Nascimento O.J.M., Lopes F.P.P.L., et al(2018).Neuroimaging findings of Zika virus-associated neurologic complications in adults[J]. AJNR Am J Neuroradiol, 39: 1967-1974.

29. Rodolphe H, Ophelie D, Sineewanlaya W, et al(2015). Biology of Zika virus infection in human skin cells[J]. J Virol, 89, 17: 8880-8896. 